\title{
SERUM 25-HYDROXY VITAMIN D, PHYSICAL ACTIVITY AND COGNITIVE FUNCTION AMONG OLDER ADULTS
}

\author{
H. Eymundsdottir ${ }^{1,2}$, M. Chang ${ }^{2,3}$, O.G. Geirsdottir ${ }^{1,2}$, P.V. Jonsson ${ }^{2,4,5}$, V. Gudnason ${ }^{4,6}$, L. Launer $^{7}$, \\ M.K. Jonsdottir ${ }^{8,9}$, A. Ramel $^{1,2}$
}

\begin{abstract}
Objective: To investigate the association between 25-hydroxy vitamin D (25OHD) and cognitive function with particular consideration of physical activity (PA) in Icelandic older adults. Design: Cross-sectional study. Setting: Iceland. Participants: Old adults aged 65-96. The final analytical sample included 4304 non-demented participants. Measurements: Serum 25OHD was categorized into deficient $(\leq 30 \mathrm{nmol} / \mathrm{L}, 8 \%)$, insufficient (31-49 nmol/L, 25\%) and normal-high levels $(>50 \mathrm{nmol} / \mathrm{L}$, 67\%). Cognitive function assessments included measurements of memory function (MF), speed of processing (SP) and executive function (EF) all categorized as low and high (divided by 50th percentile). Multivariate logistic regression analysis was used to calculate the odds ratio (OR) for having high cognitive function. Results: Serum 25OHD was positively associated with cognitive function. Adjustment for PA and other potential confounders diminished this association only partially. Compared to participants with normal-high levels of 25OHD, those with deficient levels had decreased odds for high SP (OR: 0.74, CI: 0.57-0.97), high MF (OR: 0.55; CI: 0.43-0.71) and high EF (OR: 0.76, CI: 0.57-1.0). Conclusion: Serum 25OHD below $\leq 30 \mathrm{nmol} / \mathrm{L}$ was associated with decreased odds for high cognitive function among community dwelling old adults as compared to those with $25 \mathrm{OHD}$ above $>50 \mathrm{nmol} / \mathrm{L}$. Neither PA nor other potential confounders explained the associations between 25OHD and cognitive function. Future studies should explore mechanisms and the potential clinical relevance of this relationship.
\end{abstract}

Key words: Vitamin D, memory function, speed of processing, executive function.

\section{Introduction}

Serum 25-hydroxy vitamin D (25OHD) has been recognized as crucial for maintaining calcium and phosphate homeostasis which is important for bone health (1). Further, epidemiological studies have indicated associations between low 25OHD levels and a variety of chronic illnesses including type 1 and type 2 diabetes (2), autoimmune diseases and liver disease $(3,4)$ In recent years there has been a growing interest in the potential role of vitamin $\mathrm{D}$ in cognitive function (5-9). Older adults are at a higher risk for 25OHD deficiency than younger people (10) and several studies have reported a positive correlation between $25 \mathrm{OHD}$ and cognitive function in this vulnerable group $(6,8,9)$.

1. Faculty of Food Science and Nutrition, University of Iceland, Reykjavik, Iceland. 2. The Icelandic Gerontological Research Center, The National University Hospital of Iceland, Reykjavik, Iceland; 3. Sports Science, School of Science and Engineering, Physical Activity, Physical Education, Health and Sport (PAPESH) Research Centre, Reykjavik University; 4. Faculty of Medicine, University of Iceland; 5. Department of Geriatrics, The National University Hospital of Iceland, Reykjavik, Iceland; 6 Icelandic Heart Association, Kopavogur, Iceland; 7 Laboratory of Epidemiology and Population Sciences, National Institute on Aging, National Institutes of Health (NIH), Bethesda, Maryland, USA; 8 Department of Psychology, Reykjavik University, Reykjavik; 9 Mental Health Services (Memory Clinic) Landspitali - The National University Hospital of Iceland.

Corresponding Author: Hrafnhildur Eymundsdottir, MPH. The Icelandic Gerontological Research Center, The National University Hospital of Iceland, Tungata 26, 101 Reykjavik, Iceland, (+354 8457415), E-mail hre6@hi.is
In general, aging is associated with reduction in brain tissue volume with concomitant declines in cognitive function (11). Impairment in cognitive function has also been linked to lifestyle factors, such as physical inactivity in older adults $(12,13)$. Evidence from physical activity (PA) intervention studies also indicates that PA might have a role in preserving cognitive function among older individuals (14).

Interestingly, low PA has also been associated with low levels of serum 25OHD (15), possibly related to the lack of sunlight exposure. Since several previous studies, that have investigated serum 25OHD and cognitive function, have not used statistical correction for PA $(16,17)$, it remains unclear whether the association between 25OHD and cognitive function among older adults is direct or whether it is mediated by PA.

The aim of the current study was thus to investigate the cross-sectional associations between 25OHD and cognitive function with particular consideration of the potentially mediating effects of PA using data from the Age Gene/Environment Susceptibility-Reykjavik Study (AGES-Reykjavik), a large population based cohort of older adults living at a northern latitude. 


\section{Subjects and methods}

\section{Study population}

The AGES-Reykjavik Study (AGES-RS) examined risk factors for diseases in old age, including environmental factors and genetic susceptibility, and their interactions. Briefly, the AGES-RS is a continuation of the Reykjavik Study in Iceland. The Reykjavik Study was initiated in 1967 by the Icelandic Heart Associations and included men and women born in 1907-1935 living in the Reykjavik area [18]. During 2002 - 2006, 5764 persons randomly chosen from survivors of the Reykjavik Study cohort were re-examined for the AGES-RS. Participants completed a questionnaire, underwent a clinical examination, and completed a cognitive test battery. Details on the study design and the baseline AGESRS assessments have been given elsewhere (19). The study was approved by the National Bioethics Committee in Iceland (approval VSN-00-063), The Data Protection Authority, and by the National Institute on Aging Intramural Institutional Review Board. Written informed consent was obtained from all participants.

\section{Serum 25-hydroxy vitamin D measurement}

The accredited IHA laboratory performed 25OHD measurements in batch using unfrozen serum samples and the Liaison chemiluminescence immunoassay (DiaSorin Inc, Stillwater, Minnesota). The inter-assay coefficient of variation was $<6.5 \%$ when calculated data are from measurements using a frozen serum pool as the control sample and $<12.7 \%$ when calculated data is from measurements using Liaison quality controls. Existing serum 25OHD levels were then standardized according to the international Vitamin D Standardization Program (VDSP) as previously described (20). Standardized serum $25 \mathrm{OHD}$ was used as categorized variable in statistical analyses based on Guidelines for Health Professionals from the National Institutes of Health (2014 ) (21). The cut points of serum 25OHD used in this study were as follows: deficient $(\leq 30 \mathrm{nmol} / \mathrm{L})$, insufficient (31-49 $\mathrm{nmol} / \mathrm{L})$, normal-high levels $(\geq 50 \mathrm{nmol} / \mathrm{L})$.

\section{Cognitive function assessment and dementia}

Assessment of cognitive function included nine tests, or test components, focusing on three cognitive domains, i.e., memory, processing speed and executive function. For each of the domains, a composite score was constructed based on a theoretical grouping of the tests and by converting raw scores into standardized $\mathrm{z}$ scores reflecting the distribution within the study sample. The inter-rater reliability for all tests was excellent (Spearman correlation coefficients range 0.96-0.99) (22).

The memory composite measure included the immediate and delayed-recall portions of a modified version of the California Verbal Learning Test (23). The processing speed composite measure included the Digit Symbol Substitution Test (24), the Figure Comparison Test (25) and the Stroop Test (26) Part I (reading) and Part II (color naming). The executive function composite measure included the Digits Backward Test (24), a shortened version of the CANTAB Spatial Working Memory test (27)and the Stroop Test, Part III (word-color interference). The three domains of memory, processing speed and executive function composite measures were each categorized into low (lower 50\%) and high (higher $50 \%$ ) making each domain a binary variable.

A consensus diagnosis of dementia made by a team composed of a geriatrician, neurologist, neuropsychologist, and a neuroradiologist was made according to international guidelines from the Diagnostic and Statistical Manual of Mental Disorders, Fourth Edition (28).

\section{Covariates}

A number of covariates were included in the present study including demographic, anthropometric, lifestyle, laboratory and disease-related variables:

Education was categorized into four levels (elementary school, high school, undergraduate, more than undergraduate education).

Body mass index (BMI) was calculated as $\mathrm{kg} / \mathrm{m} 2$. Smoking status was evaluated as ever vs. never smoker. Alcohol consumption was evaluated as either currently consuming vs. not consuming. Cod liver oil consumption (never, less than once a week, 1-6 times a week, daily) and multivitamins (yes/no) were assessed via questionnaire.

Leisure time physical activity was assessed by a selfreported questionnaire. Participants were asked, how many hours per week they participated in moderate/ vigorous intensity physical activity in the past 12 months. Predefined answer categories were never, rarely, weekly but $<1$ hour per week, 1-3 hours per week, 4-7 hours per week and more than 7 hours per week. In final analysis physical activity categories were combined into 1 . none, 2 . $\leq 3$ hours/week or 3. $>3$ hours/week.

Participants were instructed in advance to bring all medication they had used during the preceding twoweeks before the clinic visit and were categorized into $\leq 4$ medication vs. $\geq 5$ medication. Diabetes mellitus was defined by a physician's diagnosis of diabetes, use of diabetes medication and/or fasting blood glucose of $>7.0 \mathrm{mmol} / \mathrm{L}$. Hypertension was defined at baseline by a physician's diagnosis of hypertension, use of hypertension medications and/or blood pressure above $140 / 90 \mathrm{~mm} \mathrm{Hg}$.

A high level of depressive symptoms was classified as a score of $\geq 6$ on the 15-item Geriatric Depression Scale (29). APOE alleles were genotyped on a subsample of 2113 people using standard methods (30). APOE 
genotypes were grouped as APOE $\varepsilon 4$ carrier $(\varepsilon 3 / 4$, and $\varepsilon 4 / 4$ genotype) and APOE $\varepsilon 4$ non-carrier $(\varepsilon 2 / 2, \varepsilon 2 / 3$ and $\varepsilon 3 / 3)$.

\section{Analytical Sample}

Of the total cohort $(\mathrm{N}=5764), 5519$ had measurement of serum 25OHD, and 4699 of those had complete data on cognitive function. All subjects with dementia diagnosis $(n=180,3.1 \%)$ and APOE genotypes $\varepsilon 2 / 4(n=115,2.0 \%)$ were excluded from analysis. Participants with APOE genotypes $2 / 4$ were excluded since the allele $\varepsilon 2$ and $\varepsilon 4$ have opposite effects on the risk for cognitive impairment and dementia[30]. The final sample having complete data, included 4304 participants (Figure 1). Compared with those who were not included in the analytical sample, those included were significantly younger $(76.31 \pm 5.4$ vs. $80.42 \pm 6.7$ years, $\mathrm{p}<0.001)$ and less likely to have diabetes $(11.9 \%$ vs. $18.4 \%, \mathrm{p}<0.001)$, but the two groups did not differ significantly by gender, blood pressure or medication use.

\section{Statistical analysis}

Statistical analyses were carried out using IBM SPSS version 22.0 (SPSS, Chicago, IL, USA). Differences between participants in the 25OHD- or PA-categories were calculated using chi-square test for categorical variables and analysis of variance (ANOVA) for continuous variables (normally distributed).

The associations between 25OHD and each of the three cognitive domains were examined using logistic regression models with a different degree of statistical correction. Therefore, the $\mathrm{z}$ scores of the three domains were converted into binary variables (low and high) using the 50th percentile as a cut point. Model 1 included 25OHD categories, age, sex and education; model 2 added PA; and model 3 additionally included BMI, medication use, diabetes, hypertension, depressive symptoms, alcohol consumption and smoking status as covariates. The level of statistical significance was set at $\mathrm{p}<0.05$.

\section{Results}

Deficient and insufficient levels of 25OHD were observed in $8 \%$ and $25 \%$ of the participants, respectively, whereas $67 \%$ had normal-high levels (Table 1). All of the lifestyle-, and disease-related variables were different between the three 25OHD groups. Cod liver oil and use of multivitamin supplements were associated with higher 25OHD.

Figure 2 shows the associations between cognitive domain scores and PA stratified by 25OHD. PA was associated with higher cognitive function in the normalhigh and in the insufficient 25OHD category, however, these associations were less clear in the deficient category.
Figure 1

Flow chart

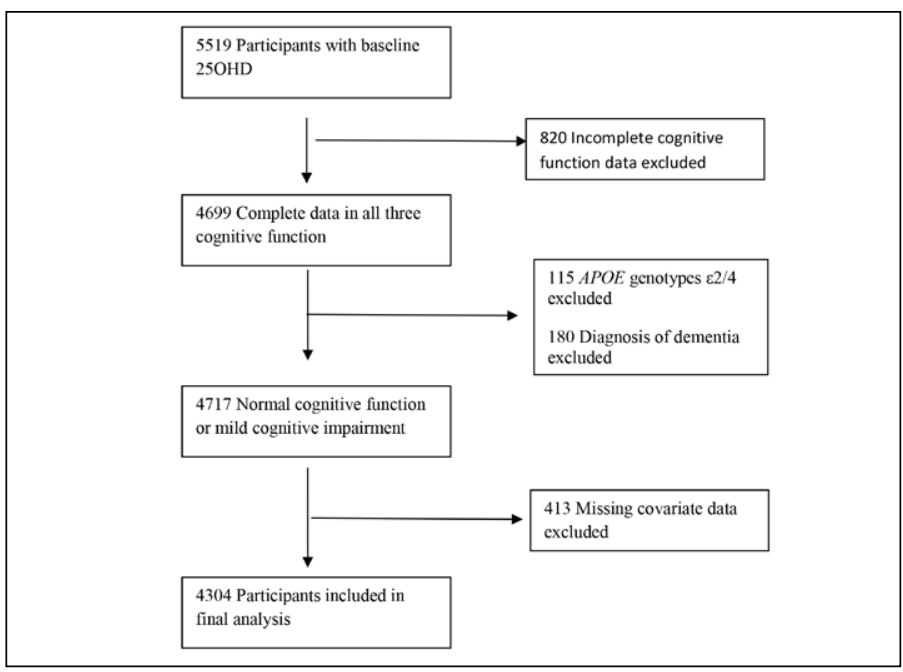

Figure 2

Standardized composite scores of each cognitive domain according to three categories of leisure time physical activity(PA); categorized as none, $\leq 3$ hours / week or $>3$ hours/week. The figures are stratified by three categories of serum 25OHD levels

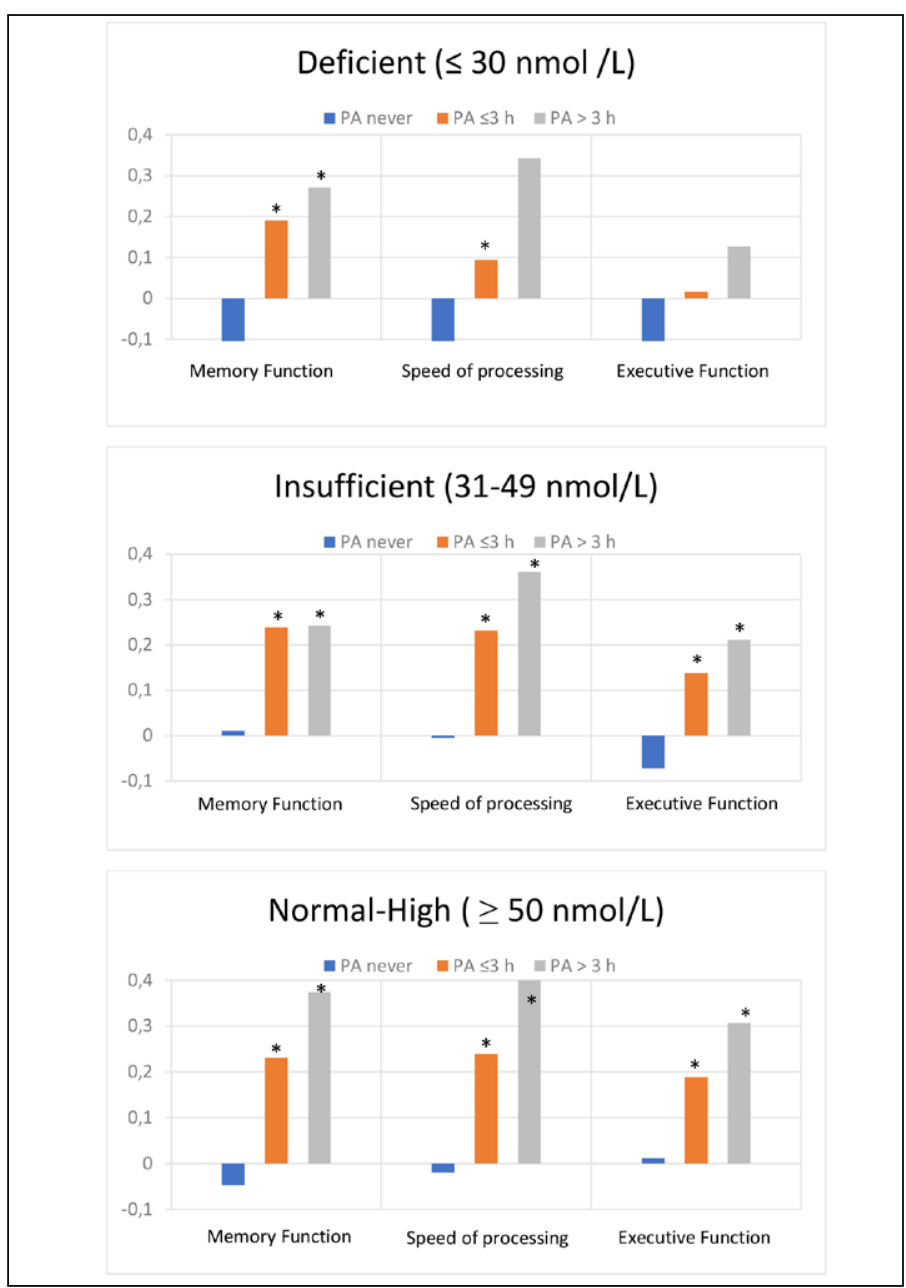

*Significantly different from the PA never group. 
Table 1

Demographic and health characteristics according to serum 25-hydroxy vitamin D concentrations among AGES/Reykjavik participants

\begin{tabular}{|c|c|c|c|}
\hline & $\begin{array}{l}\text { Deficient } \\
\leq 30 \mathrm{nmol} / \mathrm{L} \\
\mathrm{n}=331 \\
8 \%\end{array}$ & $\begin{array}{l}\text { Insufficient } \\
31-49 \text { nmol/L } \\
\mathrm{n}=1058 \\
25 \%\end{array}$ & $\begin{array}{l}\text { Normal-high } \\
\geq 50 \mathrm{nmol} / \mathrm{L} \\
\mathrm{n}=2915 \\
67 \%\end{array}$ \\
\hline Female & $69 \%$ & $61 \%$ & $54 \%$ \\
\hline Age (years) & $76.9 \pm 6.0$ & $76.5 \pm 5.5$ & $76.6 \pm 5.6$ \\
\hline Elementary education & $34 \%$ & $25 \%$ & $21 \%$ * \\
\hline $\operatorname{BMI}\left(\mathrm{kg} / \mathrm{m}^{2}\right)$ & $27.9 \pm 5.4$ & $27.7 \pm 5.4$ & $26.7 \pm 4.0^{*}$ \\
\hline \multicolumn{4}{|l|}{ Physical activity } \\
\hline None & $56 \%$ & $42 \%$ & $31 \% *$ \\
\hline$\leq 3 \mathrm{~h} /$ week & $39 \%$ & $49 \%$ & $54 \% *$ \\
\hline$>3 \mathrm{~h} /$ week & $5 \%$ & $9 \%$ & $15 \% *$ \\
\hline $\begin{array}{l}\text { Geriatric depression } \\
\text { score }(\geq 6)\end{array}$ & $8 \%$ & $7 \%$ & $5 \% *$ \\
\hline Medication $(\geq 5)$ & $45 \%$ & $41 \%$ & $39 \% *$ \\
\hline Hypertension $^{1}$ (yes) & $84 \%$ & $84 \%$ & $79 \% *$ \\
\hline Diabetes $^{2}$ (yes) & $17 \%$ & $13 \%$ & $11 \% *$ \\
\hline Smoking (yes) & $16 \%$ & $12 \%$ & $7 \% *$ \\
\hline $\begin{array}{l}\text { Daily cod liver oil } \\
\text { intake (yes) }\end{array}$ & $27 \%$ & $42 \%$ & $69 \% *$ \\
\hline Multivitamin use (yes) & $14 \%$ & $22 \%$ & $36 \% *$ \\
\hline $\begin{array}{l}\text { Alcohol consumption } \\
\text { (yes) }\end{array}$ & $49 \%$ & $59 \%$ & $66 \% *$ \\
\hline Memory function ${ }^{3}$ & $-0.24 \pm 0.9$ & $0.007 \pm 0.9$ & $0.05 \pm 0.9^{*}$ \\
\hline Speed of processing ${ }^{3}$ & $-0.21 \pm 0.9$ & $-0.05 \pm 0.9$ & $0.04 \pm 0.9^{*}$ \\
\hline Executive function ${ }^{3}$ & $-0.21 \pm 0.7$ & $-0.04 \pm 0.7$ & $0.04 \pm 0.7^{*}$ \\
\hline
\end{tabular}

Data are shown as mean \pm SD or as \%; * Significant differences between the three 25OHD categories according to chi-square test for categorical variables and analysis of variance for continuous variables; 1 . Hypertensive: systolic $\mathrm{BP}>140 \mathrm{mmHg}$, diastolic $\mathrm{BP}>90 \mathrm{mmHg}$ or medication for hypertension; 2 . Diabetes mellitus was defined by physician's diagnosis of diabetes or use of diabetes medication; 3 Standardized composite score

Results from the logistic regression analyses are shown in Table 2. We found that participants with deficient 25OHD levels were less likely (odds ratios between 0.52 - 0.76 depending on the statistical model) to have high cognitive function as compared to participants with normal-high 25OHD levels. Differences between participants with insufficient 25OHD and participants with normal-high 25OHD were not significant in the fully corrected models.

The differences in odds gradually diminished with additional covariate adjustment and thus the greatest differences between the three 25OHD categories were observed in the least corrected model 1. After additional adjustment for PA in model 2, the odds ratios for high function changed only marginally in the deficient group, e.g., from 0.58 to 0.61 for speed of processing. The changes were similar for the other domains.
In the fully corrected models the odds ratio for high function of the deficient group remained significantly lower for speed of processing (OR: 0.74, CI: 0.57-0.97) and memory function (OR:0.55, CI:0.43-0.71), but not in executive function (OR: $0.76, \mathrm{CI}: 0.58-1.00$ ).

Table 2

Odds Ratio* for high cognitive function dependent on three categories of 25-hydroxy vitamin D among AGESReykjavik participants

\begin{tabular}{|c|c|c|c|}
\hline & $\begin{array}{c}\geq 50 \mathrm{nmol} / \mathrm{L} \\
\text { (normal-high) } \\
\mathbf{n}=2915 \\
\text { OR }(95 \% \mathrm{CI})\end{array}$ & $\begin{array}{c}31-49 \mathrm{nmol} / \mathrm{L} \\
\text { (insufficient) } \\
\mathbf{n}=1058 \\
\text { OR }(95 \% \mathrm{CI})\end{array}$ & 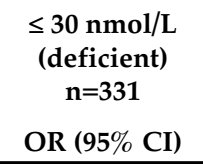 \\
\hline \multicolumn{4}{|c|}{ Speed of processing } \\
\hline Model 1 & 1.00 (ref) & $0.86(0.73-1.00)$ & $0.58(0.45-0.75)$ \\
\hline Model 2 & 1.00 (ref) & $0.88(0.75-1.03)$ & $0.61(0.47-0.77)$ \\
\hline Model 3 & 1.00 (ref) & $0.93(0.79-1.09)$ & $0.74(0.57-0.97)$ \\
\hline \multicolumn{4}{|c|}{ Executive function } \\
\hline Model 1 & 1.00 (ref) & $0.84(0.71-0.98)$ & $0.62(0.48-0.81)$ \\
\hline Model 2 & 1.00 (ref) & $0.86(0.73-1.01)$ & $0.65(0.49-0.84)$ \\
\hline Model 3 & 1.00 (ref) & $0.90(0.76-1.06)$ & $0.76(0.58-1.00)$ \\
\hline \multicolumn{4}{|c|}{ Memory function } \\
\hline Model 1 & 1.00 (ref) & $1.00(0.85-1.16)$ & $0.52(0.41-0.65)$ \\
\hline Model 2 & 1.00 (ref) & $1.02(0.87-1.19)$ & $0.55(0.43-0.71)$ \\
\hline Model 3 & 1.00 (ref) & $1.02(0.87-1.19)$ & $0.55(0.43-0.71)$ \\
\hline \multicolumn{4}{|c|}{$\begin{array}{l}\text { * Odds ratio based on multivariate logistic regression analysis; } \mathrm{CI}=\text { confidence } \\
\text { interval; OR = odds ratio; Model 1: Adjusted for age, gender, education; Model } \\
\text { 2: Adjusted for age, gender, education and physical activity; Model 3: Adjusted } \\
\text { for age, gender, education, physical activity, body mass index, depression } \\
\text { symptoms, medication, hypertension, diabetes, current smoking, and alcohol } \\
\text { consumption }\end{array}$} \\
\hline
\end{tabular}

\section{Discussion}

In this large cross-sectional study we investigated the associations between 25OHD and cognitive function among older individuals living in Iceland with attention to the potentially mediating effect of PA. We found that participants with deficient levels of 25OHD were significantly less likely to have high cognitive functioning as compared to participants with normal-high levels. PA itself was significantly associated with cognitive function, which has been reported previously. It has been suggested that PA sustains cerebral blood flow by decreasing blood pressure, lowering lipid levels and inhibiting platelet aggregation (31). However, in our study PA did not seem to mediate the associations between 25OHD and cognitive function, as inclusion of PA as covariate in statistical models barely changed the outcomes.

In general, our participants had mean levels of serum 25OHD comparable to those reported in other northern latitude countries (32-35). When looking at the characteristics of our study sample, we found that 
participants with deficient 25OHD levels had unhealthier lifestyles and poorer health compared to participants with normal-high 25OHD. They smoked more frequently, had lower engagement in PA and a higher BMI. They also had a higher incidence of depressive symptoms, diabetes, but lower educational levels (Table 1).

For the interpretation of the results it is important to consider that the above mentioned lifestyle and healthrelated variables can act as confounders. As seen in our calculations, statistical correction for these variables attenuated the relation between 25OHD and cognitive function to a certain degree. However, the association remained significant for memory function and speed of processing and borderline significant for executive function.

There is a biological basis for the role of vitamin $\mathrm{D}$ in cognitive function (5). Vitamin D receptors are found in the brain area most vulnerable to aging. They are localized in both neurons and glial cells of the brain (36). In animals, vitamin D deficiency can affect concentrations of neurotransmitters necessary for the normal function of the brain (37) . Further, animal studies have reported that vitamin D supplementation improves learning and memory impairment related to disease (38) or inflammation (39), which are important factors in the ageing process. Finally, vitamin D supplementation prevented cognitive decline in aging rats (37) in an animal model that tried to mimic the range of human 25OHD, i.e., from deficient to normal.

In the past few years several epidemiological studies have been published on vitamin $\mathrm{D}$ and cognitive function which were recently summarized in an extensive systematic review by van der Schaft et al. (40). This review included 25 cross-sectional studies on vitamin $D$ and cognitive function. In agreement with our study, the main result of the review was a statistically significant worse outcome on one or more cognitive function tests or a higher frequency of dementia with lower vitamin D levels or -intake in 18 out of $25(72 \%)$ studies. Importantly, van der Schaft et al. (40)discussed and analyzed adjustment for potential confounders to assess the relationship between vitamin $\mathrm{D}$ and cognition. There are many potential confounders, including general health, exercise and socio-economic class, of which age, level of education, BMI and gender are the most important ones (40). They found that e.g., around half of the included studies did not adjust for education or BMI. Here is where the present study adds to prior knowledge, because we could show that associations between cognitive function and 25OHD remained significant despite extensive adjustment for potential confounders. However, considering the differences in the majority of health and lifestyle variables between participants in the three 25OHD categories, we cannot exclude the possibility of residual confounding, although the inclusion of a wide range of covariates covering anthropometric, social, psychological, medical and lifestyle variables reduces such a risk.
Being cross-sectional, our study cannot determine whether low 25OHD is a cause or a consequence of low cognitive function. Longitudinal study designs can address this question. The above mentioned review (39) included five prospective cohort studies showing that participants with low 25OHD had faster cognitive decline than those with high 25OHD. This was also shown in a more recently published prospective cohort study by Karakis et al. (41). However, as prospective cohort studies are still sensitive to the effects of confounding, and thus placebo-controlled randomized clinical trials are needed to confirm results obtained from both cross-sectional and prospective cohort studies

\section{Strengths and limitations}

It is a strength of the current study that we used detailed cognitive assessment that allowed us to examine specific cognitive domains in relation to 25OHD. Also, several health-related, socioeconomic, and lifestyle variables were available for our sample, so we could adjust for number of important confounders in the statistical analysis. Finally, the final sample size was large, comprising 4304 participants. However, it is an inherent limitation of this study like all other crosssectional studies that one cannot disentangle cause and effect.

\section{Conclusion}

In our sample of community dwelling old adults, participants with deficient 25OHD were less likely to have high memory function or high speed of processing. PA was associated with high cognitive function, however it did not explain the associations between 25OHD and cognitive function.

Acknowledgments: Funding: This work was supported by The Foundation of St. Josef's Hospital in cooperation with The Icelandic Gerontological Research Center National University Hospital of Iceland. The AGES-RS study was supported by the National Institutes of Health (Intramural Research Programs of the National Institute of Aging and the National Eye Institute, ZIAEY00401), National Institutes of Health contract number N01-AG-1-2100, the Icelandic Heart Association, and the Icelandic Parliament.

Conflict of Interest: The authors declare that they have no conflict of interest.

Ethical approval: AGES-Reykjavik was approved by the National Bioethics Committee in Iceland that acts as the institutional review board for the IHA (approval number VSN-00-063) and the National Institute on Aging Intramural Institutional Review Board. A multistage consent is obtained for AGES-Reykjavik to cover participation and access to administrative records. Release of data for analysis is governed by rules created by these bodies to protect the privacy of Icelandic participants.

Informed consent: Informed consent was obtained from all individual participants included in study.

\section{References}

1. Dusso, A.S., A.J. Brown, and E. Slatopolsky, Vitamin D. Am J Physiol Renal Physiol, 2005. 289(1): p. F8-28.

2. Afzal, S., S.E. Bojesen, and B.G. Nordestgaard, Low 25-hydroxyvitamin D and risk of type 2 diabetes: a prospective cohort study and metaanalysis. Clin 
Chem, 2013. 59(2): p. 381-91.

3. Wang, J., et al., Meta-analysis of the association between vitamin D and autoimmune thyroid disease. Nutrients, 2015. 7(4): p. 2485-98.

4. Iruzubieta, P., et al., Vitamin D deficiency in chronic liver disease. World J Hepatol, 2014. 6(12): p. 901-15.

5. Buell, J.S. and B. Dawson-Hughes, Vitamin D and neurocognitive dysfunction: preventing "D"ecline? Mol Aspects Med, 2008. 29(6): p. 415-22.

6. Wilkins, C.H., et al., Vitamin D deficiency is associated with low mood and worse cognitive performance in older adults. Am J Geriatr Psychiatry, 2006. 14(12): p. 1032-40.

7. Przybelski, R.J. and N.C. Binkley, Is vitamin D important for preserving cognition? A positive correlation of serum 25-hydroxyvitamin D concentration with cognitive function. Arch Biochem Biophys, 2007. 460(2): p $202-5$

8. Slinin, Y., et al., Association between serum $25(\mathrm{OH})$ vitamin $\mathrm{D}$ and the risk of cognitive decline in older women. J Gerontol A Biol Sci Med Sci, 2012. 67(10): p. $1092-8$.

9. Miller, J.W., et al., Vitamin D Status and Rates of Cognitive Decline in a Multiethnic Cohort of Older Adults. JAMA Neurol, 2015. 72(11): p. 1295-303.

10. Ramel, A., et al., Vitamin D deficiency and nutritional status in elderly hospitalized subjects in Iceland. Public Health Nutrition, 2009. 12(7): p. 10015.

11. Ikram, M.A., et al., Brain tissue volumes in relation to cognitive function and risk of dementia. Neurobiol Aging, 2010. 31(3): p. 378-86.

12. Chang, M., et al., The effect of midlife physical activity on cognitive function among older adults: AGES--Reykjavik Study. J Gerontol A Biol Sci Med Sci, 2010. 65(12): p. 1369-74

13. Colcombe, S.J., et al., Aerobic fitness reduces brain tissue loss in aging humans. J Gerontol A Biological Science and Medical Science, 2003. 58(2): p. 176-80.

14. Kirk-Sanchez, N.J. and E.L. McGough, Physical exercise and cognitive performance in the elderly: current perspectives. Clin Interv Aging, 2014. 9: p. 51-62.

15. Brock, K., et al., Low vitamin D status is associated with physical inactivity, obesity and low vitamin D intake in a large US sample of healthy middleaged men and women. J Steroid Biochem Mol Biol, 2010. 121(1-2): p. 462-6.

16. Etgen, T., et al., Vitamin D deficiency, cognitive impairment and dementia: a systematic review and meta-analysis. Dement Geriatr Cogn Disord, 2012. 33(5): p. 297-305.

17. Annweiler, C., et al., Vitamin D and cognitive performance in adults: a systematic review. Eur J Neurol, 2009. 16(10): p. 1083-9.

18. Bjornsson, H.B., OJ. Davidson, D et al. , Health survey in the Reykjavik areawomen (No. abc 24 (XXIV)):The Icelandic Heart Association. 1982.

19. Harris, T.B., et al., Age, Gene/Environment Susceptibility-Reykjavik Study: multidisciplinary applied phenomics. Am J Epidemiol, 2007. 165(9): p. 107687.

20. Cashman, K.D., et al., Vitamin D deficiency in Europe: pandemic? Am J Clin Nutr, 2016. 103(4): p. 1033-44.

21. health, N.i.o. Vitamin D. 2016, February 11; Available from: https://ods. od.nih.gov/factsheets/VitaminD-HealthProfessional/.

22. Qiu, C., et al., Diabetes, markers of brain pathology and cognitive function: the Age, Gene/Environment Susceptibility-Reykjavik Study. Ann Neurol, 2014. 75(1): p. 138-46
23. Delis DC, K.J., Kaplan E, Ober BA., California Verbal Learning Test ManualAdult Version (Research Edition). 1987, New York Psychological Corporation 24. Wechsler, D., Adult Intelligence Scale 1955, New York: Psycological Corporation.

25. Salthouse, T.A. and A.W. Kersten, Decomposing adult age differences in symbol arithmetic. Mem Cognit, 1993. 21(5): p. 699-710.

26. Stroop, J., Studies of interference in serial verbal reactions. Journal of Experimental Psyocology 1933. 121(1): p. 15-21.

27. Robbins, T.W., et al., Cambridge Neuropsychological Test Automated Battery (CANTAB): a factor analytic study of a large sample of normal elderly volunteers. Dementia, 1994. 5(5): p. 266-81.

28. Sheehan, D.V., et al., The Mini-International Neuropsychiatric Interview (M.I.N.I.): the development and validation of a structured diagnostic psychiatric interview for DSM-IV and ICD-10. J Clin Psychiatry, 1998. 59 Suppl 20: p. 22-33;quiz 34-57.

29. Yesavage, J.A., et al., Development and validation of a geriatric depression screening scale: a preliminary report. J Psychiatr Res, 1982. 17(1): p. 37-49.

30. Corder, E.H., et al., Gene dose of apolipoprotein E type 4 allele and the risk of Alzheimer's disease in late onset families. Science, 1993. 261(5123): p. 921-3.

31. Rogers, R.L., J.S. Meyer, and K.F. Mortel, After reaching retirement age physical activity sustains cerebral perfusion and cognition. J Am Geriatr Soc 1990. 38(2): p. 123-8

32. Bates, B., Lennox, A., Prentice, A., Bates, C., and P. Page, Nicholson, S. and Swan, G., National Diet and Nutrition Survey: Results from Years 1, 2, 3 and 4 (combined) of the Rolling Programme (2008/2009 - 2011/2012). 2014, Public Health England.

33. Snijder, M.B., et al., Adiposity in relation to vitamin D status and parathyroid hormone levels: a population-based study in older men and women. J Clin Endocrinol Metab, 2005. 90(7): p. 4119-23.

34. Andersen, R., et al., Teenage girls and elderly women living in northern Europe have low winter vitamin D status. Eur J Clin Nutr, 2005. 59(4): p. 53341.

35. Holick, M.F., High prevalence of vitamin D inadequacy and implications for health. Mayo Clin Proc, 2006. 81(3): p. 353-73.

36. Eyles, D.W., et al., Distribution of the vitamin D receptor and 1 alphahydroxylase in human brain. J Chem Neuroanat, 2005. 29(1): p. 21-30.

37. Latimer, C.S., et al., Vitamin D prevents cognitive decline and enhances hippocampal synaptic function in aging rats. Proc Natl Acad Sci U S A, 2014 111(41): p. E4359-66.

38. Moghadamnia, A.A., et al., Vitamin D Improves Learning and Memory Impairment in Streptozotocin-Induced Diabetic Mice. Arch Iran Med, 2015. 18(6): p. 362-6.

39. Tian, A., et al., Vitamin D improves cognitive function and modulates Th17/T reg cell balance after hepatectomy in mice. Inflammation, 2015. 38(2): p. 500-9.

40. van der Schaft, J., et al., The association between vitamin D and cognition: a systematic review. Ageing Res Rev, 2013. 12(4): p. 1013-23.

41. Karakis, I., et al., Association of Serum Vitamin D with the Risk of Inciden Dementia and Subclinical Indices of Brain Aging: The Framingham Heart Study. J Alzheimers Dis, 2016. 51(2): p. 451-61. 